\title{
EL MARKETING COMO HERRAMIENTA DE GESTIÓN EN LO PROFESIONAL Y PERSONAL
}

\author{
Jorge V. Mayurí Barrón (*) \\ E-mail: jvmayuri@hotmail.com
}

\begin{abstract}
RESUMEN
La globalización de los mercados, hoy más que nunca, genera continuos cambios en gustos y preferencias por parte del consumidor, cambios que a su vez generan cambios en la oferta y demanda, haciendo que la presión competitiva cada día sea más intensa, por lo que el especialista en Marketing de hoy debe trabajar con un modelo que le permita lograr los objetivos de la empresa al final de todo un proceso continuo y de calidad.

Dicho modelo se inicia previa investigación de mercados, con la identificación de la cultura y de las necesidades de los consumidores del mercado meta, con el propósito de desarrollar satisfactores de aquellas necesidades para que, a través de una segmentación de mercados y un estudio del comportamiento del consumidor, tengamos la información que permitirá al área de ventas colocar el producto con las mejores ventajas dentro del mercado, además de fidelizar al cliente.

En forma paralela es importante determinar las estrategias de marketing que permitan lograr un mejor posicionamiento a través de las variables del Marketing (precio, producto, comunicación y distribución) que contribuyan al mejoramiento del Plan de Marketing, con lo que se realizaría una gestión eficiente del Marketing Operativo que es el pilar del Marketing Estratégico.
\end{abstract}

Palabras clave: Cultura, necesidades, oferta y demanda, mercado meta, comportamiento del consumidor, participación de mercado, plan de marketing.

\section{ABSTRACT}

Today more than ever, the globalization of markets, produces continuous changes in choices and preferences by consumers. These changes cause changes in supply and demand, making everyday the competitive pressure to be more intense, so that the marketing specialist of today, must work with a marketing model, that allows him at the end of a qualified and continuous process to achieve the targets of the company.

(*) Doctor en Educación, Magíster en Administración con Mención en Gestión Empresarial. Licenciado en Administración de Empresas con Post Grado en Derecho Aéreo y Espacial. Profesor Asociado de la Facultad de Ciencias Administrativas de la UNMSM, y Profesor de Post Grado en USMP, UNFV, UNSCH, UNCP. Capacitador Empresarial. 
The above mentioned model starts previous to a market research, identifying the culture and needs of consumers of the target market with the purpose of developing satisfiers from those needs, so that through a market segmentation and a study of consumer behavior, we can have the information that will allow the sales area to place the product with the best advantages in the market to achieve the customer loyalty.

In a parallel way it is important to determine the marketing strategies that allow a better positioning through marketing variables, price, product, communication and distribution for the improvement of the marketing plan, to achieve an efficient management of the Operational marketing which is the cornerstone of strategic marketing.

Keywords: Culture, Needs, supply and demand, target market, consumer behavior, market share, marketing plan.

\section{INTRODUCCIÓN}

Es evidente que el mercado cambia constantemente, ello obedece a que los gustos y preferencias de los clientes y consumidores así lo hacen. Por esto, el especialista en mercados debe de estar atento a todos estos cambios a fin de adecuar sus estrategias de Marketing a estas variantes, ya que si no lo hace, la competencia siempre estará alerta, desplazando a toda aquella organización que no se adapte a este mercado. Hoy en día la competencia es muy intensa y se requiere de especialistas en Marketing capaces de adaptarse a todas estas nuevas tendencias de mercado, a fin de garantizar el crecimiento sostenido de su organización.

La labor del especialista en Marketing empieza con el análisis cultural del mercado al cual pretende ingresar, comprendiendo la cultura ${ }^{1}$, esto incluye costumbres, prácticas, códigos, normas y reglas de la manera de ser, vestimenta, religión, rituales, normas de comportamiento y sistemas de creencias.

Así, es importante que conozca los productos culturales de cada cultura, tales como:

- Ceremonia. Sistema de varios ritos seleccionados con una ocasión o evento.

- Creencia. Interpretación de un fenómeno concreto.

- Cuento. Relato de un hecho imaginario.

- Hazaña. Relato histórico que describe los logros singulares de un grupo y sus líderes, por regla general en términos heroicos.
- Héroes/heroínas. Personas legitimadas por la organización que sirven de modelo para la conducta de otros.

- Historia. Relato basado en hechos verídicos, en ocasiones combinación de verdades y ficciones.

- Lenguaje. Una forma o manera particular de sonidos y signos escritos usada por los miembros de un grupo para trasmitir significados entre sí.

- Leyenda. Relato de un hecho magnífico que pasa de una generación a otra, basado en la historia, pero embellecido con toques imaginarios.

- Metáfora. Resumen de palabras usado para captar una visión o reforzar valores, antiguos o nuevos.

- Mito. Relato dramático de hechos imaginarios, normalmente usado para explicar el origen o la transformación de algo. Además creencia incuestionable en los beneficios prácticos de ciertas técnicas y conductas que no está sustentada en hechos.

- Ritos. Serie de actividades relativamente elaboradas, dramáticas y proyectadas que consolidan diversas formas de expresiones culturales en un solo evento, realizadas por medio de interacciones sociales, normalmente para beneficio de un público.

- Ritual. Serie de técnicas y comportamientos estandarizados y detallados que manejan angustias pero que rara vez producen las consecuencias técnicas de gran importancia práctica que se pretenden.

1 Entiéndase por cultura al conjunto de todas las formas, los modelos o los patrones, explícitos o implícitos, a través de los cuales una sociedad regula el comportamiento de las personas que la conforman. 
- Símbolo. Cualquier objeto, acto, hecho, calidad o relación que sirve de vehículo para transmitir un significado, generalmente mediante la representación de otra cosa.

- Valores. Actitudes que rigen la existencia y sirve de guías de vida para la conducta.

Una vez analizados estos productos culturales, el siguiente paso es realizar una investigación de mercados a través de sistemas de investigación de mercados, que vendrían a ser el Diseño sistemático, la Recolección, el Análisis y la Presentación de la información y de los descubrimientos relevantes acerca de una situación de marketing específica a la que se enfrenta la empresa.

La investigación de mercados tiene un gran campo de acción:

- Investigación corporativa y de negocios / económica.

- Características y tendencias en la industria / en el mercado.

- Estudio de adquisición y diversificación.

- Análisis de porción de mercado.

- Estudio interno de los empleados (moral, comunicación, otros).

- Precios.

- Análisis de costos.

- Análisis de utilidades.

- Elasticidad de precios.

- Análisis de demanda (potencial absoluto, potencial actual y pronóstico de ventas).

- Análisis de precios competitivos.
- Producto.

- Desarrollo y prueba de concepto.

- Generación y prueba de nombre de marca.

- Prueba piloto de mercado.

- Prueba de productos existentes.

- Estudio de diseño de empaque.

- Estudio de productos competitivos.

- Distribución.

- Estudio de ubicación de plantas / bodegas.

- Estudio de funcionamiento de canales.

- Estudio de cobertura de canales.

- Estudio internacional y de exportación.

- Promoción.

- Investigación de motivación.

- Investigación de medios.

- Investigación de textos publicitarios.

- Efectividad de la publicidad.

- Estudio de la publicidad de la competencia.

- Estudio de imagen pública.

- Estudio de remuneración de la fuerza de ventas.

- Estudio de cuotas de la fuerza de ventas.

- Estructura del territorio de la fuerza de ventas.

- Estudio de premios, cupones, otros.

- Conducta de compra.

- Preferencia de marcas.

- Actitud en cuanto a marcas.

- Satisfacción respecto al producto.

- Comportamiento de compra.

- Intención de compra.

- Conciencia de marca.

- Estudio de segmentación.

Gráfico N. ${ }^{\circ}$ 1. Investigación del Marketing

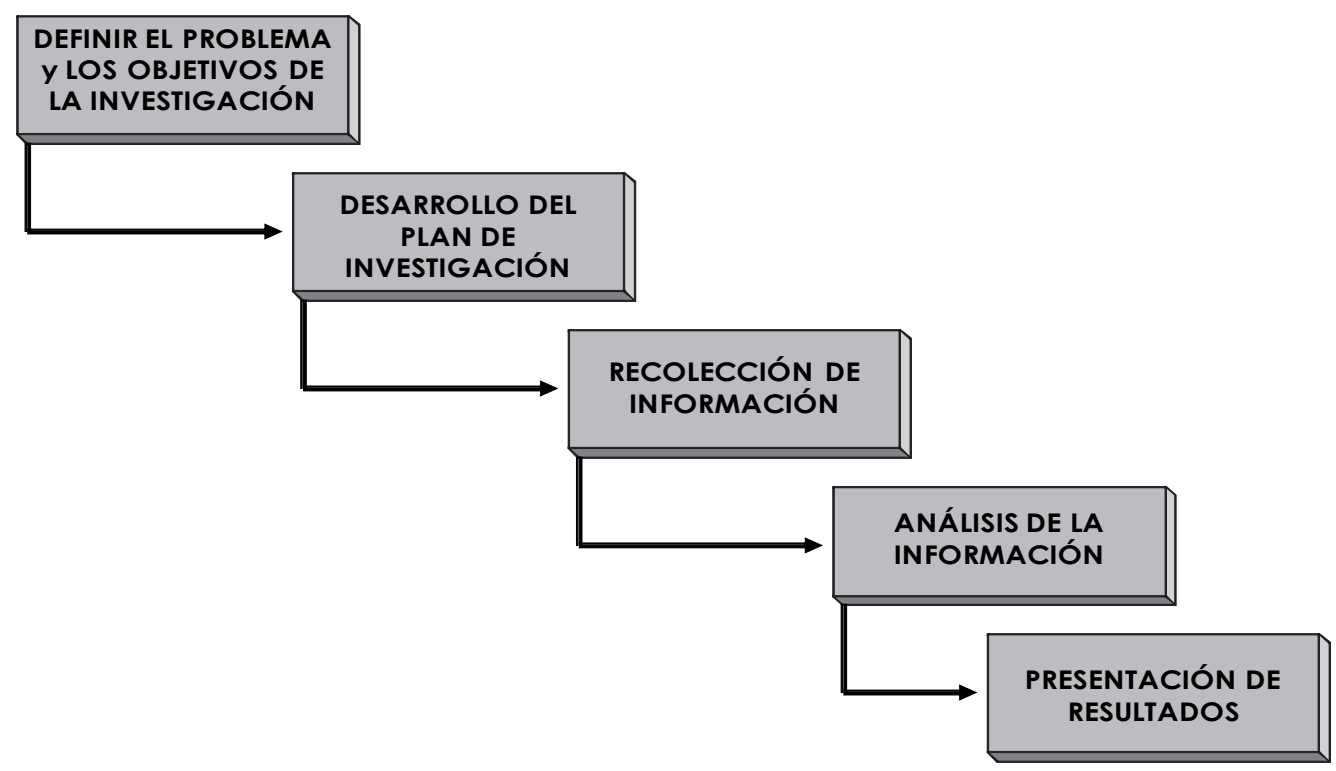




\section{NECESIDADES DEL CONSUMIDOR}

Esta investigación, entre otros puntos, nos permite detectar las necesidades del consumidor en el Marketing y en los Recursos Humanos.

Una necesidad para una persona es una sensación de carencia unida al deseo de satisfacerla. Por ejemplo, la sed, el hambre y el frío son sensaciones que indican la necesidad de agua, alimento y calor, respectivamente. Para poder entender mejor esto podemos recurrir a la famosa Jerarquía de Necesidades de Abraham Maslow.

La escala de las necesidades de Maslow se describe a menudo como una pirámide que consta de cinco niveles: los cuatro primeros niveles pueden ser agrupados como "necesidades de déficit" (deficit needs o $D$-needs); al nivel superior lo denominó "autorrealización", "motivación de crecimiento", o "necesidad de ser" (being needs o B-needs). La diferencia estriba en que mientras las necesidades de déficit pueden ser satisfechas, la necesidad de ser es una fuerza impelente continua.

La idea básica de esta jerarquía es que las necesidades más altas ocupan nuestra atención sólo cuando se han satisfecho las necesidades inferiores de la pirámide. Las fuerzas de crecimiento dan lugar a un movimiento ascendente en la jerarquía, mientras que las fuerzas regresivas empujan las necesidades prepotentes hacia abajo en la jerarquía.

Una vez detectadas las necesidades del consumidor hay que desarrollar aquellos satisfactores que permitan satisfacer estas necesidades. Los satisfactores son productos y/o servicios que, a través de sus características o atributos, satisfacen plenamente al consumidor.

Según la pirámide de Maslow dispondríamos de los siguientes tipos de necesidades (ver Gráfico N. ${ }^{\circ} 2$ ):

\section{Necesidades básicas}

Son necesidades fisiológicas básicas para mantener la homeostasis (referente a la salud). Dentro de estas, las más evidentes son:

- Necesidad de respirar, beber agua, y alimentarse.

- Necesidad de mantener el equilibrio del pH y la temperatura corporal.

- Necesidad de dormir, descansar y eliminar los desechos.

- Necesidad de evitar el dolor.

- Necesidad de amar y ser amado

\section{Necesidades de seguridad y protección}

Éstas surgen cuando las necesidades fisiológicas se mantienen compensadas. Son las necesidades de sentirse seguro y protegido, incluso desarrollar ciertos límites en cuanto al orden. Dentro de ellas se encuentran:

- Seguridad física y de salud.

- Seguridad de empleo, de ingresos y recursos.

- Seguridad moral, familiar y de propiedad privada.

Gráfico $\mathrm{N}^{\circ}{ }^{2}$. Pirámide de Maslow

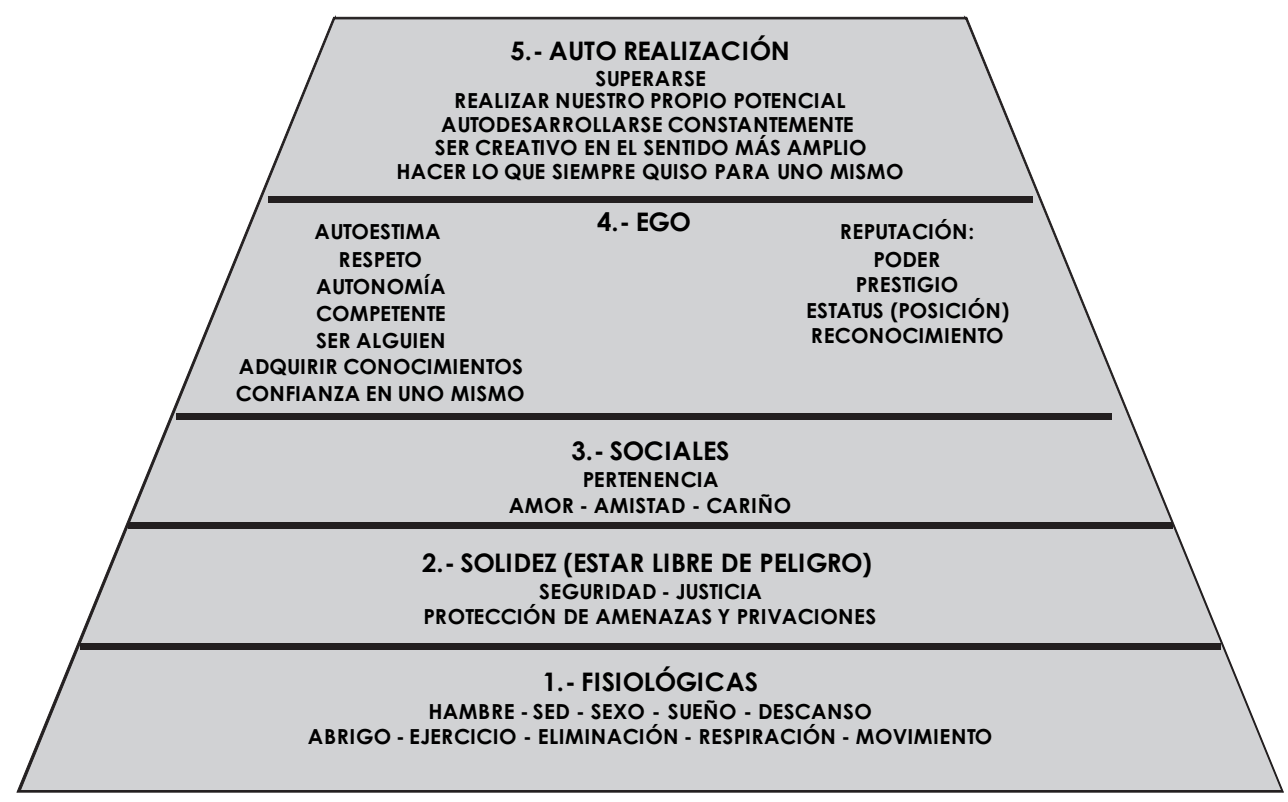

Gestión en el Tercer Milenio, Rev. de Investigación de la Fac. de Ciencias Administrativas, UNMSM (Vol. 13, No 25, Lima, julio2010). 


\section{Necesidades de afiliación y afecto}

Están relacionadas con el desarrollo afectivo del individuo, son las necesidades de:

- Asociación

- Participación

- Aceptación

Se satisfacen mediante las funciones de servicios y prestaciones que incluyen actividades deportivas, culturales y recreativas. El ser humano por naturaleza siente la necesidad de relacionarse, ser parte de una comunidad, de agruparse en familias, con amistades o en organizaciones sociales.

Entre estas necesidades se encuentran la amistad, el compañerismo, el afecto y el amor, que se forman a partir del esquema social.

\section{Necesidades de estima}

Maslow describió dos tipos de necesidades de estima, una alta y otra baja.

- La estima alta concierne a la necesidad del respeto a uno mismo, e incluye sentimientos tales como confianza, competencia, maestría, logros, independencia y libertad.

- La estima baja concierne al respeto de las demás personas: la necesidad de atención, aprecio, reconocimiento, reputación, estatus, dignidad, fama, gloria, e incluso dominio.

La merma de estas necesidades se refleja en una baja autoestima y el complejo de inferioridad.

\section{Autorrealización o autoactualización}

Este último nivel es algo diferente y Maslow utilizó varios términos para denominarlo: "motivación de crecimiento", "necesidad de ser" y "autorrealización".

Son las necesidades más elevadas, se hallan en la cima de la jerarquía y, a través de su satisfacción, se encuentra un sentido a la vida mediante el desarrollo potencial de una actividad. Se llega a ésta cuando todos los niveles anteriores han sido alcanzados y completados, al menos, hasta cierto punto.

\section{SEGMENTACIÓN DEL MERCADO}

Cuando ya se establecieron los satisfactores para el mercado es necesario segmentarlo. Entiéndase por segmentación al proceso de dividir el mercado total de bienes y servicios en grupos más pequeños, de modo que los miembros de cada grupo sean semejantes respecto a los factores que influyen en la demanda.

La segmentación de mercado es el proceso de dividir un mercado en grupos uniformes más pequeños que tengan características y necesidades semejantes. Esto no está arbitrariamente impuesto sino que se deriva del reconocimiento de que el total de mercado está hecho de subgrupos llamados segmentos.

Estos segmentos son grupos homogéneos (por ejemplo, las personas en un segmento son similares en sus actitudes sobre ciertas variables). Debido a esta similitud dentro de cada grupo es probable que respondan de modo similar a determinadas estrategias de marketing. Es decir, probablemente tendrán las mismas reacciones acerca del marketing mix de un determinado producto, vendido a un determinado precio, distribuido en un modo determinado y promocionado de una forma dada.

Los requisitos para una buena segmentación son:

- Homogeneidad en el segmento.

- Heterogeneidad entre segmentos.

- Estabilidad de segmentos.

- Los segmentos deben ser identificables y medibles.

- Los segmentos deben ser accesibles y manejables.

- Los segmentos deben ser lo suficientemente grandes como para ser rentables.

Las variables utilizadas para segmentación incluyen:

\section{- Variables geográficas}

- Región del mundo o del país.

- Tamaño del país.

- Clima.

\section{- Variables demográficas}

- Edad.

- Género.

- Orientación sexual.

- Tamaño de la familia.

- Ciclo de vida familiar.

- Ingresos.

- Profesión.

- Nivel educativo. 
- Estatus socioeconómico.

- Religión.

- Nacionalidad.

\section{- Variables psicográficas}

- Personalidad.

- Estilo de vida.

- Valores.

- Actitudes.

\section{- Variables de comportamiento}

- Búsqueda del beneficio.

- Tasa de utilización del producto.

- Fidelidad a la marca.

- Utilización del producto final.

- Nivel de listo para consumir.

- Unidad de toma de decisión.

Cuando muchas variables se combinan para proporcionar un conocimiento profundo del segmento, se le conoce como segmentación profunda. Cuando se da suficiente información para crear una imagen clara del miembro típico del segmento, se llama perfil del comprador. Una técnica estadística utilizada habitualmente en determinar un perfil es el cluster análysis (análisis de grupo)

Los beneficios de la segmentación de mercados pueden ser:

- Identificar las necesidades más específicas para los submercados.

- Focalizar mejor la estrategia de marketing.

- Optimizar el uso de los recursos empresariales de:

- Marketing.

- Producción.

- Logística.

- Toma de decisiones.

- Hacer publicidad más efectiva.

- Identificar un nicho propio donde no tenga competencia directa.

- Aumentar las posibilidades de crecer rápidamente en segmentos del mercado sin competidores.

La segmentación genera muchas ventajas hoy en día tales como:

- Al segmentar el mercado se investiga cuáles son las necesidades de cada segmento para satisfacerlas.
- Al trabajar con segmentos se puede hacer un mejor uso de los recursos de marketing.

Pero también genera desventajas como:

- Son con respecto a los costos y cobertura del mercado.

- La producción resulta más costosa, por no ser en masa.

- Mayor costo de inventarios por la diversidad de productos.

- Incremento de inversión publicitaria.

- La gerencia debe planear y realizar varios programas de marketing incrementando los gastos administrativos.

\section{COMPORTAMIENTO DEL CONSUMIDOR}

Identificadas las necesidades, desarrollados los satisfactores y segmentado el mercado debemos iniciar un estudio del comportamiento del consumidor, que es la conducta que los consumidores tienen cuando buscan, compran, usan, evalúan y desechan productos y servicios que esperan que satisfagan sus necesidades.

Su estudio tiene que ver con las razones por las que compran los consumidores, cuándo, dónde, y cómo compran, y con qué frecuencia lo hacen.

Es importante para el gerente y el investigador de marketing la determinación de los propósitos y objetivos del estudio (cualitativo o cuantitativo).

Basado en los propósitos del estudio:

- Si es información descriptiva, el estudio es cuantitativo.

- Si es la obtención de nuevas ideas, el estudio es cualitativo.

Para el diseño de una investigación cualitativa existen tres técnicas:

- Entrevista a profundidad.

- Grupos focales.

- Técnicas proyectivas.

Para el diseño de una investigación cuantitativa existen tres técnicas:

- Investigación observacional.

- Experimentación.

- Encuestas.

Es en este momento que recién estamos en condiciones de pasar la información al Departamento de Ventas de la empresa a fin de que diseñe sus estrategias apropiadas para capturar el mercado. Toda la información que de acá en 


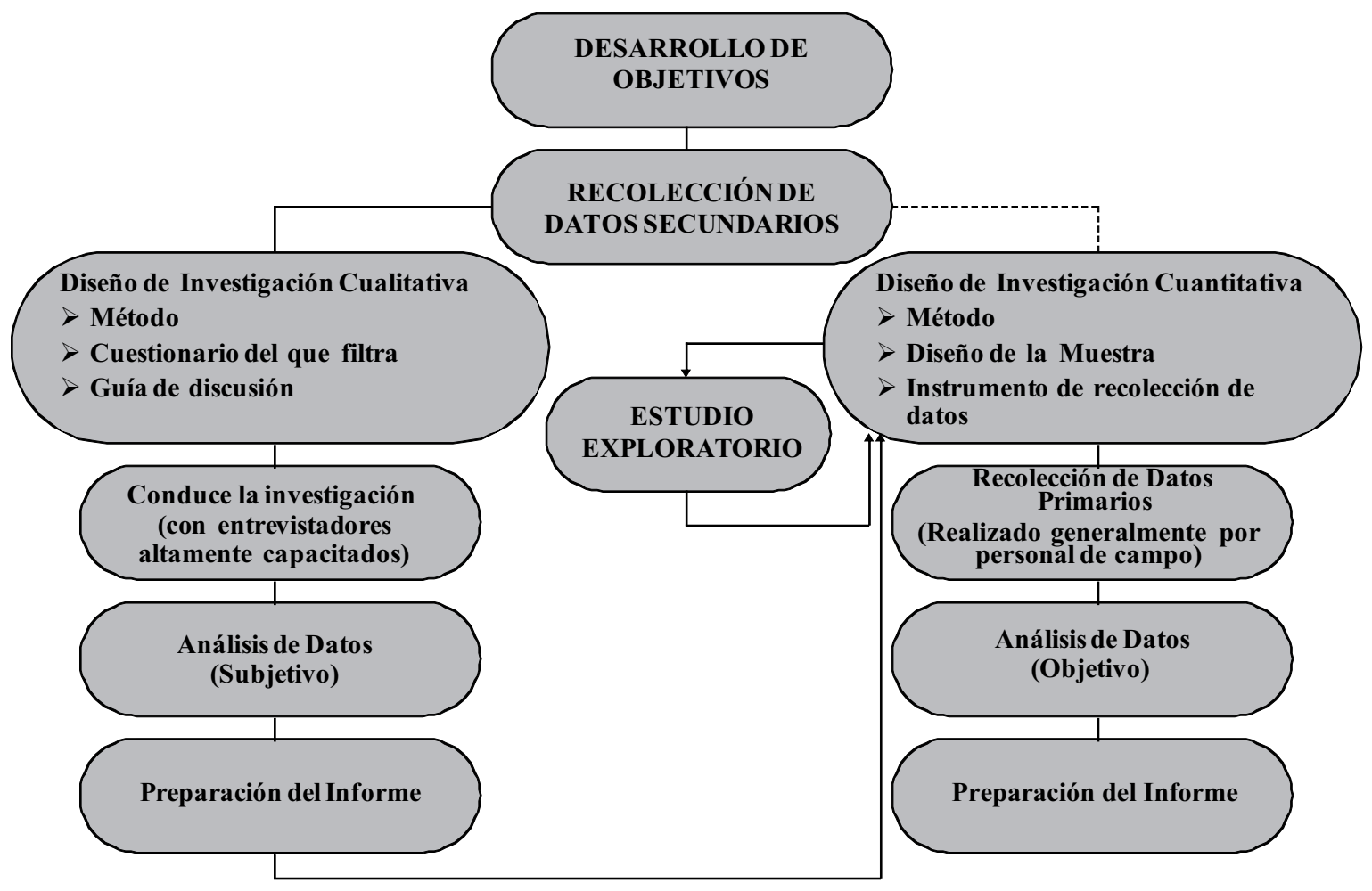

adelante se levante permitirá que postventa pueda desarrollar competencias que permitan fidelizar al consumidor y/o cliente. Recordemos que hoy en día dicha fidelización es cada día más difícil debido a la oferta existente en el mercado que, a través del mejoramiento de sus productos y/o servicios, busca generar una mejor participación de mercado.

\section{MARKETING MIX}

Paralelo a todo este proceso antes visto, tenemos que trabajar con el famoso mix comercial, que son las cuatro variables del marketing: precio, producto, comunicación y distribución, variables totalmente importantes, ya que de fallar cualquiera de ellas nuestro producto o servicio llegaría al mercado en desventaja.

\section{Producto}

Cualquier bien, servicio, idea, persona, lugar, organización o institución que se ofrezca en un mercado para su adquisición, uso o consumo y que satisfaga una necesidad.

La política de producto incluye el estudio de cuatro elementos fundamentales:
- La cartera de productos.

- La diferenciación de productos.

- La marca.

- La presentación.

\section{Precio}

Es el valor de intercambio del producto determinado por la utilidad o la satisfacción derivada de la compra, y el uso o el consumo del producto.

Es el elemento del mix que se fija más a corto plazo y con el que la empresa puede adaptarse rápidamente según la competencia, costo, etc. Se distingue del resto de los elementos del marketing mix porque es el único que genera ingresos, mientras que los demás elementos generan costos.

Para determinar el precio, la empresa deberá tener en cuenta lo siguiente:

- Los costos de producción, distribución, etc.

- El margen de ganancia que se desea obtener.

- Los elementos del entorno, principalmente la competencia.

- Las estrategias de Marketing adoptadas.

- Los objetivos establecidos. 


\section{Distribución o plaza}

Elemento del mix que utilizamos para conseguir que un producto llegue satisfactoriamente al cliente. Cuatro elementos configuran la política de distribución:

- Canales de distribución. Los agentes implicados en el proceso de mover los productos, desde el proveedor hasta el consumidor.

- Planificación de la distribución. La toma de decisiones para implantar una sistemática de cómo hacer llegar los productos a los consumidores y los agentes que intervienen (mayoristas, minoristas).

- Distribución física. Formas de transporte, niveles de stock, almacenes, localización de plantas y agentes utilizados.

- Merchandising. Técnicas y acciones que se llevan a cabo en el punto de venta. Consiste en la disposición y la presentación del producto al establecimiento, así como de la publicidad y la promoción en el punto de venta.

\section{Comunicación o promoción}

La comunicación persigue difundir un mensaje y que éste tenga una respuesta del público objetivo al que va destinado. Los objetivos principales de la comunicación son:

- Comunicar las características del producto.

- Comunicar los beneficios del producto.

- Que se recuerde o se compre la marca/producto.

La comunicación no es sólo publicidad. Los diferentes instrumentos que configuran el mix de comunicación son los siguientes:

- La publicidad.

- Las relaciones públicas.

- La venta personal.

- La promoción de ventas.

- El Marketing directo.

\section{POSICIONAMIENTO}

En el marketing se utilizan las estrategias con la finalidad de generar un Posicionamiento. Se llama posicionamiento a la referencia del lugar que en la percepción mental de un cliente o consumidor tiene una marca, lo que constituye la principal diferencia que existe entre ésta y su competencia. También se conoce así a la capacidad del producto de alienar al consumidor.
Por todos los medios se debe evitar que el posicionamiento ocurra sin la debida planificación, razón por la que se emplean técnicas consistentes en la planeación y comunicación de estímulos diversos para la construcción de la imagen e identidad deseada para la marca a instaurar en la subjetividad del consumidor, lo cual es totalmente agresivo y contra la voluntad de las personas.

El posicionamiento es un principio fundamental que muestra su esencia y filosofía, ya que lo que se hace con el producto no es el fin sino el medio por el cual se accede y trabaja con la mente del consumidor. Se posiciona un producto en la mente del consumidor; así, lo que ocurre en el mercado es consecuencia de lo que sucede en la subjetividad de cada individuo en el proceso de conocimiento, consideración y uso de la oferta; de allí que el posicionamiento hoy se encuentre estrechamente vinculado al concepto rector de propuesta de valor, que considera el diseño integral de la oferta a fin de hacer la demanda sostenible en horizontes de tiempo más amplios.

Reposicionamiento es cambiar la posición que el producto o servicio tiene en la mente del cliente, o bien en ocasiones recordar uno que ya había olvidado.

La capacidad de identificar una oportunidad de posicionamiento es un buen test para conocer las habilidades de un experto en marketing. Las estrategias exitosas de posicionamiento se traducen en la identificación de una ventaja competitiva por parte de un producto.

Las bases más comunes para construir una estrategia de posicionamiento de producto son:

- Posicionamiento sobre soluciones, beneficios o necesidades específicas.

- Posicionamiento sobre el uso determinado de categorías.

- Posicionamiento sobre ocasiones de uso específicas.

- Posicionamiento contra otro producto.

- Posicionamiento a través de disociación por tipo de producto.

Generalmente existen tres tipos de conceptos de posicionamiento:

- Posiciones funcionales.

- Resolver problemas.

- Proporcionar beneficios a los consumidores. 
- Posiciones simbólicas.

- Incremento de la propia imagen.

- Identificación del ego.

- Pertenencia y significado social.

- Filiación afectiva.

- Posiciones experienciales.

- Proporcionar estimulación sensorial.

- Proporcionar estimulación cognitiva.

Encontrar un posicionamiento se ve facilitado por una técnica gráfica llamada mapeado perceptual y varias técnicas de investigación y técnicas estadísticas como escalado multidimensional, análisis factorial, análisis conjunto y análisis lógico.

Generalmente, el proceso de posicionamiento de producto comprende:

- Identificar productos competidores.

- Identificar los atributos (también llamados dimensiones) que definen el "espacio" del producto.

- Recoger información de una muestra de consumidores sobre sus percepciones de los atributos relevantes de cada producto.

- Determinar la cuota de cada producto que ocupa la mente de los consumidores.
- Determinar la localización actual de cada producto en el espacio del mercado.

- Determinar las combinaciones favoritas de atributos de quienes constituyen los mercados objetivos (respecto a un vector ideal).

- Examinar la concordancia entre:

- Las posiciones de productos competidores.

- La posición de tu producto.

- La posición de un vector ideal.

- Seleccionar la posición óptima.

Recordemos que la mente acepta aquello que encaja en los conocimientos y experiencias anteriores por lo que es difícil cambiar una opinión cuando ésta ya se ha formado; como los consumidores están saturados de información sobre productos y servicios es importante que la comunicación sea eficiente.

\section{PLAN DE MARKETING}

Con toda la información ya trabajada elaboramos un documento que llamamos Plan de Marketing, el que debe hacerse por cada uno de los productos o servicios principales que tenemos en nuestra organización.

Gráfico N. ${ }^{\circ}$ 4. Marketing Operativo y Marketing Estratégico

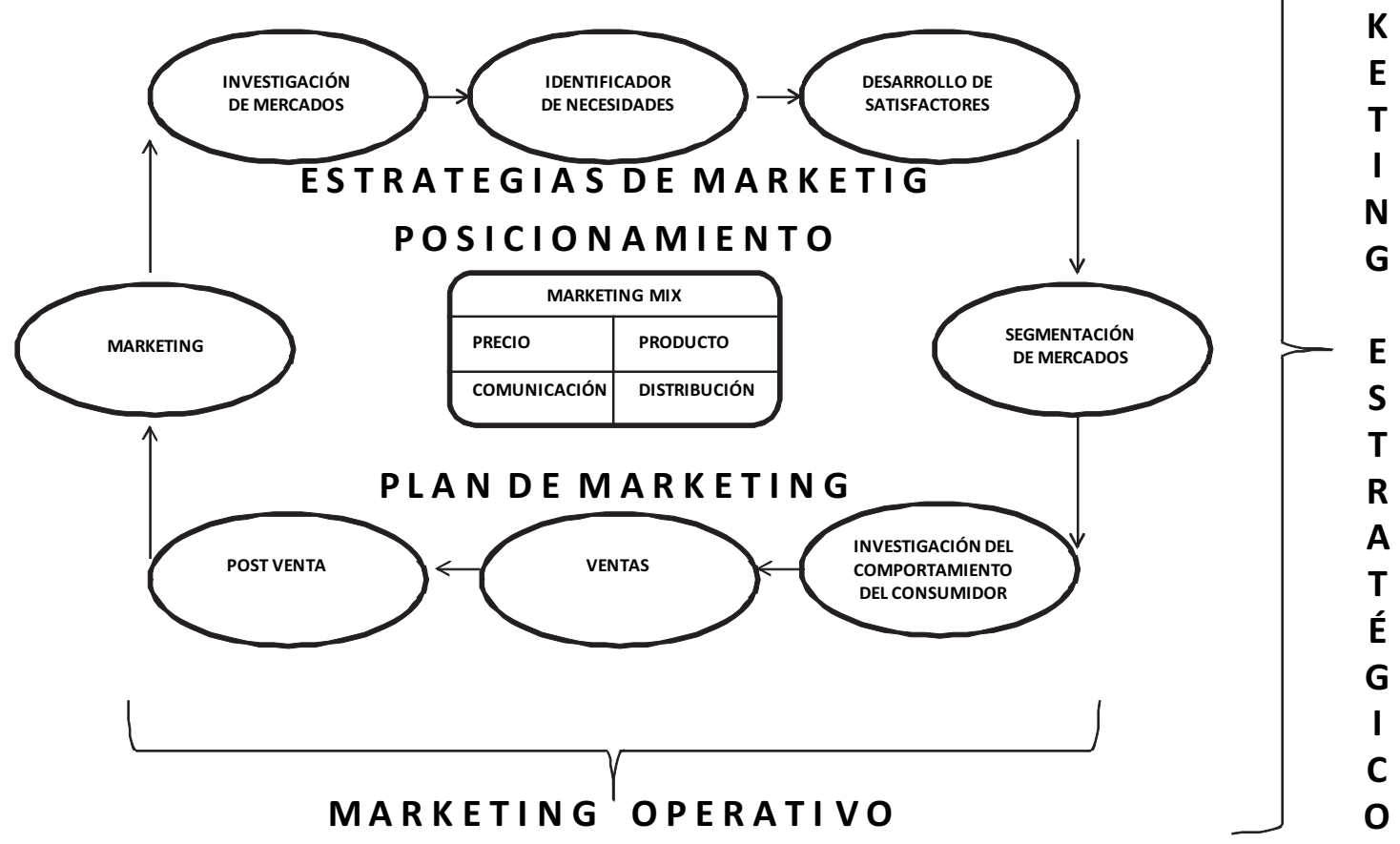


En este plan se determinan todas las acciones de marketing que vamos a hacer el próximo año con la finalidad de ganar un mejor posicionamiento que nos permita incrementar nuestra participación de mercado.

Todo lo anteriormente dicho está ubicado dentro de lo que conocemos como el Marketing Operativo o Táctico, que es el pilar principal del Marketing Estratégico.

Entiéndase por Marketing Operativo a la gestión voluntarista de conquista de los mercados existentes, cuyo horizonte de acción se sitúa en el corto y mediano plazo

Marketing Estratégico es el proceso adoptado por una organización que tiene una orientación mercado y cuyo objetivo consiste en obtener un rendimiento económico más elevado que el del mercado, a través de una política continua de creación de productos y servicios que aportan a los usuarios un valor superior al de las ofertas de la competencia.

\section{CONCLUSIONES}

1. El campo de acción del marketing es total tanto en personas naturales como jurídicas.

2. Por la intensa presión competitiva, las empresas hoy en día tienen que ser más efectivas y eficaces.

3. El marketing es una herramienta de gestión que, llevada en forma profesional, puede ayudar al logro de los objetivos empresariales y personales.

4. Las necesidades son carencias de algo, por lo que es función del marketing encontrar ese algo que, en otras palabras, es un satisfactor.

5. El especialista en marketing debe trabajar armónicamente con las variables del marketing mix: precio, producto, comunicación y distribución.

6. El especialista en marketing debe seleccionar la mejor estrategia general que permita lograr el posicionamiento de los productos o servicios de la empresa, con el propósito de incrementar la participación de ésta en el mercado.

7. La labor del especialista en marketing va en función del mercado y para el mercado, por eso debe estar atento a los cambios del mismo, a fin de adaptar sus estrategias a ello.
8. El Plan de Marketing es un documento que ayuda a la gestión de marketing del próximo año.

9. El marketing no solamente sirve para las empresas sino que todo su modelo puede ser adaptado a las personas que, si lo aplican en forma correcta, podrían llegar a ser más competitivas en el mercado.

\section{BIBLIOGRAFÍA}

\section{Libros}

ARELLANO CUEVA, ROLANDO. (2002). Comportamiento del Consumidor, Enfoque América Latina. México D.F., Mc Graw Hill Interamericana Editores S.A. de CV.

ASSAEL, HENRY (1999). Comportamiento del Consumidor. Sexta Edición. México D.F., International Thomson Editores.

DAVID, FRED (2008). Conceptos de Administración Estratégica. Novena Edición. México, Pearson Educación de México S.A. de CV.

KOTLER, PHILIP; CÁMARA, DIONICIO; GRANDE, IDELFONSO; CRUZ, IGNACIO (2000). Dirección de Marketing: edición del milenio. Décima Edición. Madrid, Pearson Educación S.A.

KOTLER, PHILIP; KELLER, KEVIN LANE (2006). Dirección de Marketing. Décimasegunda Edición. México D.F., Pearson Educación de México S.A. de CV.

KOTLER, PHILIP (2007). Marketing. México D.F., Prentice Hall.

KOTLER, PHILIP (2008). Fundamentos de Marketing. México D.F., Pearson Educación.

LAMBIN, JEAN-JACQUES (1997). Marketing Estratégico. Tercera Edición. España, Mc Graw-Hill / Interamericana de España SAU.

LEE KIM, HYUN-SOOK (2009). Marketing Internacional: teoría y 50 casos. México D.F., CENGAGE Learning.

MAYORGA, DAVID (2005). Marketing Estratégico en la empresa peruana. Lima, Universidad del Pacífico, Centro de Investigación.

MCCARTHY, E. JEROME; PERREAULT, WILLIAM D. JR. (2000). Marketing: un enfoque global. Décimo Tercera Edición. México D.F., Mc Graw Hill Interamericana Editores S.A. de CV. 
MULLINS, JOHN W. (2007). Administración del Marketing. México D.F., Mc Graw-Hill Interamericana.

PORTER, MICHAEL E. (1996). Ventaja Competitiva. México D.F., Compañía Editorial Continental SA. de CV.

PRIDE M., WILLIAM; FERREL, O. C. (1996). Marketing: conceptos y estrategias. Novena Edición. México D.F., Mc Graw Hill.

RICO, RUBÉN ROBERTO (2008). Export Marketing. Buenos Aires, Prentice Hall.

RUSSELL, J. THOMAS; LANE, W. RONALD (1994). Publicidad. Décima Segunda Edición. México D.F., Prentice Hall Hispanoamericana SA.

SANTESMASES MESTRE, MIGUEL (1997). Marketing, Conceptos y Estrategias. Tercera Edición. Madrid, Ediciones Pirámide SA.

SCHIFFMAN, LEÓN G. ; KANUT, LESLIE LAZAR (1997). Comportamiento del Consumidor. Quinta Edición. México D.F., Prentice Hall Hispanoamérica SA.

SOLOMÓN R., MICHAEL (1997). Comportamiento del Consumidor. Tercera Edición. México D.F., Prentice Hall Hispanoamericana SA.

STANTON, WILLIAM J. (2007). Fundamentos de Marketing. México D.F, Mc Graw-Hill.

SUN TZU (2009). "El arte de la guerra”. Barcelona, Brontes.
VILDÓSOLA BASAY, MARIO (2009). Gerencia de Marketing. Lima, Fondo Editorial de la UNMSM.

WALKER, ORVILLE C. (2005). Marketing Estratégico. México D.F., Mc Graw-Hill.

ZEITHAML, VALARIE A.; JOBITNER, MARY JO. (2002). Marketing de Servicios: un enfoque de integración de cliente a empresa. Segunda Edición. México, Mc Graw Hill Interamericana Editores SA. de CV.

ZIKMUND, WILLIAM G. (1998). Investigación de Mercados. Sexta Edición. México, Prentice Hall Hispanoamericana SA.

\section{Internet}

http://es.wikipedia.org/wiki/Estrategia_de_marketing http://es.wikipedia.org/wiki/Investigaci\%C3\%B3n_de mercados

http://es.wikipedia.org/wiki/Marketing

http://es.wikipedia.org/wiki/Mezcla_de mercadotecnia

http://es.wikipedia.org/wiki/Pir\%C3\%A1mide_de_Maslow

http://es.wikipedia.org/wiki/Posicionamiento

http://es.wikipedia.org/wiki/Segmentaci\%C3\%B3n_ de_mercado 